\section{A signal detection analysis of the effects of hearing on the visual recognition of two-digit numbers}

\author{
EDWARD M. BAKER and JOHN R. SCHUCK \\ Bowling Green State University, Bowling Green, Ohio 43403
}

A signal detection measure of visual recognition was used to assess the visual recognition of two-digit numbers in three different conditions: (1) voicing of the number as it was presented visually; (2) voicing of a different number than the one presented; and ( 3 ) no voicing. The three treatment means were significantly different, with matched voicing resulting in the best performance and no voicing, in the worst. There was no evidence that the treatments differentially affected response criteria.

Smith (1965) reported that visual recognition was facilitated by a perceptual interaction with hearing. In his study, each $O$ was required to recognize a tachistoscopically presented list of 25 two-digit numbers. Each visual presentation was accompanied by a voicing by the $\mathrm{E}$. There were 5 matched trials during which $E$ voiced the same number that was presented visually. On the 20 mismatched trials, E voiced a number other than the one presented visually. The voicing began prior to and terminated after the visual stimulus. The visual exposure duration used for each $O$ was the median estimate of the thresholds for three numbers as obtained by the method of limits. The Os were instructed to respond only when they were at least $50 \%$ sure of being correct. It was found that Os had significantly better visual recognition in the matched condition than they had in the mismatched condition.

This outcome has important implications for theories dealing with the way humans process information simultaneously presented in two sensory modalities. However, there was a possible artifact in Smith's procedure. In order to correct for the possible tendency of each $O$ to try to achieve more hits in the matched trials by simply repeating the number that he heard $E$ voice, Smith adjusted the number of correct trials obtained in the matched condition with the proportion of times that $O$ responded on the mismatched trials with the same number that had just been voiced. If that proportion were $25 \%$, for example, then the total number correct in the matched condition was reduced by $25 \%$.

This approach to response bias is based on the traditional high-threshold model of the sensory system and no longer appears valid in the light of a great deal of recent research by signal detection theorists (Green \& Swets, 1966; Swets, 1964). The signal detection model considers O's errors to be based on valid sensory information and not the result of guessing. Regardless of O's sensitivity, the obtained error rate can be high or low, depending on the value of being correct and the cost of being wrong. Loveless, Brebner, and Hamilton (1970), in their review of bisensory stimulation research, have criticized studies such as Smith's from a signal detection viewpoint. Simultaneous stimulation in a second modality can raise $O$ 's hit rate not only through sensory interaction, but by changing $O$ 's decision criterion so that he becomes more likely to respond that a signal was present during the observation interval.

The purpose of this experiment, therefore, was to assess Smith's (1965) results, using a signal detection analysis of the recognition task. In addition to the matched and mismatched conditions, a no-voicing condition was included in order to determine if mismatching actually inhibits visual recognition.

\section{METHOD \\ Observers}

Six male undergraduate students between the ages of 20 and 24 served as Os. Each was paid $\$ 10$ for serving in the experiment.

\section{Apparatus}

Visual stimuli, two-digit numbers, were displayed on two numerical neon-glow readout tubes (National Electronics) that were set $1 / 3$ in. apart. The numbers were visible at eye level behind a 13/4-in.-diam circular opening, centered on a 27-in.-high $x$ 18-in.-wide Masonite board that was painted flat black. A $1 / 2-$ in.-wide white border surrounded the opening. $A$ green ready light was set 6 in. above the opening. Neutral density filters (Kodak) were placed between the Masonite board and the readout tubes to control the brightness of the numbers. Os were in a darkened acoustical chamber (Industrial
Acoustics) and sat $3 \mathrm{ft}$ from the display in a chair with adjustable height and headrest.

$\mathbf{E}$ was in an adjoining chamber where he could activate the number display with either a voice key or a pushbutton. A Hunter timer controlled the duration of time a number appeared on the display. Voicing was done by $E$ over a Fanon-Masco intercom (Model FX-2B). E's intercom was always in the talk position and O's was always in the listen position. $O$ 's chamber had four response buttons with a light above each to indicate to $O$ which button he had pressed. O's response indicator lights were wired to four identical lights in E's chamber and could be terminated only by E's reset button.

\section{Procedure}

The same numbers, 47, 54, 79, and 95, were used for all Os. Pilot data obtained from six $O$ s by the method of limits indicated that there were no systematic differences in the recognizability of these numbers on the readout tubes. One of the four numbers, randomly chosen for each $O$, was designated as the signal (S). The other three were designated as nonsignal $(\mathrm{N})$. By depressing one of the four response buttons, $O$ 's task was to report whether the signal number was presented. The buttons were labeled, from left to right, "yes-sure," "yes-not sure," "no-not sure," and "no-sure."

There were three experimental conditions: (1) Matched (M). Each visual presentation of a $\mathrm{S}$ or $\mathrm{N}$ number was accompanied by a matched voicing of that number. There were 300 presentations per $0,120 \mathrm{~S}$ and 180 N. (2) Mismatched (MM). Each visual presentation of a $S$ or $\mathbf{N}$ number was accompanied by the voicing of a number other than the one visually presented. There were $\mathbf{3 0 0}$ presentations per $\mathrm{O}, 120 \mathrm{~S}$ and $180 \mathrm{~N}$. (3) No Voicing (NV). In this situation, no voicing accompanied the visual presentation of $\mathbf{S}$ or $\mathbf{N}$ number. There were 240 presentations per $O$, $96 \mathrm{~S}$ and $144 \mathrm{~N}$.

Each $O$ received a different random sequence of the $\mathbf{8 4 0}$ presentations of $S$ and $N$ at an a priori $P(S)=.40$. In order to reduce the possible tendency of $\boldsymbol{O}$ to say the signal when he heard it, the conditional probability of the visual signal's being presented, given that it had been voiced, was held at .50. O was told that both the conditional and a priori probability of the signal was about .50 . He was shown his signal number on the display but was not told what or how many $\mathbf{N}$ numbers there were. Each $\mathbf{N}$ 
number appeared equally often, both visually and voiced.

There were three 2-h blocks of 420 trials for each $O$. The data from the first block were not used in the data analysis since this block of trials was used to train $O$ in the use of the rating scale, to get the correct chair adjustment for complete vision of the number display, and to ascertain the exposure duration for each $O$, which would establish his overall hit rate between $70 \%$ and $95 \%$ for the three conditions. The first estimation of exposure duration was obtained by the method of limits. After that, the rating method was used. In order to keep the exposure durations close to those used by Smith, and, also, to insure that the visual stimulus would terminate before voicing ended, neutral density filters of 5.0 (for five $O s$ ) and 4.5 (for one $O$ ) were used. This resulted in a range of display durations over $O s$ of 50-100 msec. Once the exposure duration was established for an $O$, it remained constant throughout the experimental trials.

A trial lasted for about 5 sec. Following a protocol sheet for each 0 , which listed the sequence of stimulus presentation, $E$ pressed the button that preset the number display and turned on O's ready light. Two seconds later, $E$ activated the display with the voice key or with the pushbutton if it was a NV trial. When the visual stimulus and ready light terminated, $O$ responded. $E$ recorded $O$ 's response and then turned off both sets of response-indicator lights. Approximately $2 \mathrm{sec}$ after $O$ responded, $E$ began a new trial by setting another number on the display and activating the ready light. $O$ could indicate problems to $E$ with a "trouble" button, which flashed a light in E's chamber, but the button was never used during the experiment.

After each block of 35 trials, $O$ took a 2-min rest inside the chamber. There was a 15-min rest period outside of the chamber after 210 trials. Whenever $O$ had been out of the chamber, 15 extra warm-up trials were given but these data were not used in the statistical analysis.

Hit and false alarm rates were calculated as follows:

Hit rates. $\mathrm{P}(\mathrm{y} / \mathrm{SS})$-proportion of yes responses, given that the sigmal number was presented visually and vocally (matched condition). $\mathrm{P}(\mathrm{y} / \mathrm{Sn})$-proportion of yes responses, given that the signal number was presented visually but a nonsignal number was voiced (mismatched condition). $P(y / S)$-proportion of yes responses, given that the signal number was presented visually with no voicing (no-voicing condition).

False alarm rates. $\mathrm{P}(\mathrm{y} / \mathrm{NN})$-proportion of yes responses, given that the same nonsignal number was presented visually and vocally (matched condition). $P(y / N n)$-proportion of yes responses, given that a nonsignal number was presented visually but a different nonsignal number was voiced ( $m$ is matched condition). $P(y / N s)$-proportion of yes responses, given that a nonsignal number was presented visually but the signal number was voiced (mismatched condition). $P(y / N)$-proportion of yes responses, given that a nonsignal number was presented visually with no voicing (no-voicing condition).

\section{RESULTS AND DISCUSSION}

The first analysis determined whether $O$ tended to repeat what he heard $E$ say, rather than responding to the visual stimuli. This was accomplished by calculating the two proportions, $P(y / N n)$ and $P(y / N s)$, for all Os. The mean $\mathrm{P}(\mathrm{y} / \mathrm{Nn})$ was .142 and the mean $P(y / N s)$ was .103. A two-tailed $t$ test of the difference between correlated measures was not significant $(t=1.74 ; p>.05)$. This indicated that $O$ s were not responding "yes" more often when the $S$ numbers were voiced than when the $N$ numbers were voiced. Therefore, the false alarm rate for the MM condition was obtained by pooling $P(y / N n)$ and $P\left(y / N_{s}\right)$ for each $O$

Following the procedure described by Green and Swets (1966), the rating responses were used to obtain receiver operating characteristic (ROC) curves for each $O$ in each condition. The area of the unit square under the $R O C$ curve in each condition was taken as a measure of the recognizability of the $S$ number. The mean areas were .813 , .749 , and .696 , for the $M, M M$, and NV conditions, respectively. A one-way randomized-blocks analysis of variance indicated a significant difference among treatments $(F=15.85 ; \mathrm{df}=2 / 10 ; p<.001)$.

The Newman-Keuls post hoc procedure was used to test the significance of the differences among these mean areas (Kirk, 1968). The value of the critical difference for means two steps apart was .047; for means three steps apart the value was $.058(\alpha=.05 ; \mathrm{d} f=10)$. All differences exceeded the appropriate critical values.

An analysis of response bias was performed to determine if voicing produced a change in O's criterion, i.e., a change in willingness to respond positively that the signal was present. The bias measure used was that suggested by Hodos (1970). This measure yields a value of zero when the $R O C$ point falls on the negative diagonal of the unit square. Points to the left of the negative diagonal yield positive values, indicating an unwillingness to respond positively. Points to the right of the negative diagonal yield negative values, indicating a willingness to respond positively. Bias values were calculated from the middle data point for each individual ROC function. The mean values were $22.85,22.20$, and 22.38 , for the $M, M M$, and $N V$ conditions, respectively. A randomized-blocks analysis of variance indicated no significant differences among these means $(F<1)$, supporting the notion that Os maintained the same response criterion in all treatment conditions.

In summary, matched voicing produced significantly better recognition than did mismatched voicing. Mismatching, however, was better than no voicing at all. There was no indication that the three experimental treatments had differential effects upon $O$ 's response criterion.

This kind of outcome suggests that voicing, whether matched or mismatched, improves performance through a signaling or alerting function. The more important conclusion, however, is that matched voicing has an additional effect upon recomition. Matched voicing of a number facilitates recognition of that number in a way not entirely due to the alerting properties of voicing. Our study eliminates the ambiguity associated with Smith's (1965) conclusions by ruling out the possibility that matched voicing causes $O$ to bias his report.

\section{REFERENCES}

GREEN, D. M., \& SWETS, J. A. Signal detection theory and psychophysics. New Youk: Wiley, 1966.

HODOS, W. Nonparametric index of response bias for use in detection and recognition experiments. Psychological Bulletin, 1970, 74, 351-354.

KIRK, R. E. Experimental design: Procedures for the behavioral sciences. Belmont, Calif: Brooks-Cole, 1968.

LOVELESS, N, E., BREBNER, J., \& HAMILTON, P. Bisensory presentation of information. Psychological Bulletin, $1970,73,161-199$.

SMITH, $\mathbf{W}$. M. Visual recognition: Facilitation of seeing by hearing. Psy chonomic Science, 1965, 2, 157-158.

SWETS, J. A. (Ed.) Signal detection theory and recognition by human observers: Contemporary readings. New York: wiley, 1964.

(Accepted for publication June 5, 1972.) 